\title{
TTR
}

Traduction, terminologie, rédaction

\section{Corinne Wecksteen et Ahmed El Kaladi, dirs. La Traductologie dans tous ses états. Mélanges en l'honneur de Michel Ballard. Arras, Artois Presses Université, 2007, 284 p.}

\section{Stéphanie Roesler}

Volume 22, numéro 1, 1er semestre 2009

La traduction au Japon

Translation in Japan

URI : https://id.erudit.org/iderudit/044792ar

DOI : https://doi.org/10.7202/044792ar

Aller au sommaire du numéro

Éditeur(s)

Association canadienne de traductologie

ISSN

0835-8443 (imprimé)

1708-2188 (numérique)

Découvrir la revue

Citer ce compte rendu

Roesler, S. (2009). Compte rendu de [Corinne Wecksteen et Ahmed El Kaladi, dirs. La Traductologie dans tous ses états. Mélanges en l'honneur de Michel Ballard. Arras, Artois Presses Université, 2007, 284 p.] TTR, 22(1), 275-281. https://doi.org/10.7202/044792ar

Tous droits réservés @ $@$ TTR: traduction, terminologie, rédaction — Les auteurs, 2010
Ce document est protégé par la loi sur le droit d'auteur. L'utilisation des services d'Érudit (y compris la reproduction) est assujettie à sa politique d'utilisation que vous pouvez consulter en ligne.

https://apropos.erudit.org/fr/usagers/politique-dutilisation/ 
FINNEGAN, Ruth (1992). Oral Traditions and the Verbal Arts. London and New York, Routledge.

FIRTH, J.R. (1956). “Linguistic analysis and translation.” In For Roman Jakobson: Essays on the Occasion of His Sixtieth Birthday. Hague, Mouton, pp. 133-139.

NICOLAS, L. (1994). L'algonquin au XVII siècle. Diane Daviault, dir. Sainte-Foy, Presses de l'Université du Québec.

\section{Brian Mossop YorK UNIVERSITY}

\section{Corinne Wecksteen et Ahmed E1 Kaladi, dirs. La Traductologie dans tous ses états. Mélanges en l'bonneur de Michel Ballard. Arras, Artois Presses Université, 2007, 284 p.}

La Traductologie dans tous ses états est un volume d'articles offerts par ses amis et collègues à Michel Ballard, qui a décidé de partir à la retraite à la fin de l'année universitaire 2006-2007. Le titre, en jouant sur l'expression "être dans tous ses états ", témoigne d'une part de l'émotion suscitée par le départ du célèbre traductologue, laissant " la traductologie orpheline d'un de ses plus ardents défenseurs et promoteurs » ( Avant-propos », p. 8.), et reflète la diversité des études traductologiques que Michel Ballard a pu inspirer. Les articles du volume, écrits par des auteurs de huit pays différents, abordent des sujets variés et consistent aussi bien en réflexions théoriques, études sur un corpus d'un aspect traductologique particulier ou encore en analyses de caractère linguistique. Si les éditeurs nont pas opéré de classement afin de permettre au lecteur de cheminer à travers l'ouvrage à sa guise, quelques lignes directrices se dégagent dans les thèmes abordés.

Quelques articles sont consacrés à des questions théoriques. Ainsi, celui de Claude Bocquet, intitulé "Traduire les textes nobles, traduire les textes ignobles : une seule ou deux méthodes? ", qui revient sur le jugement de valeur selon lequel seule la traduction des textes littéraires serait noble car son objet est noble, jugement qui a poussé certains auteurs et 
traductologues à reléguer la traduction non noble à une question purement technique et à établir des méthodes de traduction différentes selon le type de texte. Dans le domaine théorique toujours, Michael Mariaule a choisi de mettre "l'adaptation à l'épreuve de la traduction » et de définir plus précisément la notion d'adaptation. Quoique l'adaptation soit souvent définie par rapport à la traduction en traductologie, elle reste une notion polymorphe et polyvalente. Mariaule éclaire sa réflexion en discutant les idées de Georges L. Bastin afin de montrer que traduction et adaptation ne sopposent pas réellement, chacune participant de l'autre.

Plusieurs articles sont situés dans la mouvance du cultural turn qui s'est opéré en traductologie dès le milieu des années 1970 et traitent des rapports entre traduction et culture. Ainsi, dans son article « Comment analyser la traduction interculturelle? », Lieven D'hulst propose de faire dialoguer la traduction intertextuelle et la " traduction interculturelle ", notion qu'il définit théoriquement et qu'il étudie à travers l'exemple des littératures antillaises contemporaines. Il propose de développer une méthodologie appropriée à l'étude intégrée de la traduction, ainsi que d'aller vers l'analyse de la traduction interculturelle, qui ne passe pas nécessairement par une opération langagière, ce qui suppose de donner à la traductologie une dimension interdisciplinaire.

Ce n'est pas la traduction interculturelle mais la traduction transculturelle sur laquelle s'interroge Christine Raguet dans son article « $\mathrm{Y}$ a-t-il des limites à la traduction transculturelle? ». Elle propose donc de franchir les multiples limites imposées à la littérature et à la traduction dans le cadre de la traduction transculturelle, d'envisager des stratégies traductives permettant de combler la distance culturelle entre l'espace d'origine et l'espace d'arrivée et de se familiariser avec l'inquiétante étrangeté de l'Autre. Elle aborde le cas particulier des cultures exotiques, où se posent par exemple les problèmes de la traduction de l'oralité, de la créolisation ou de l'utilisation de langages cryptés.

Ahmed El Kaladi a choisi quant à lui d'examiner les problèmes de traduction que pose la littérature issue de l'acculturation dans son article "Au-delà de l'écran des mots. 
Interculturalité et traduction ». Pour ce faire, il examine la traduction de La Nuit sacrée de Tahar Ben Jelloun en arabe et en anglais. Ses objectifs sont de montrer que cette littérature a des spécificités que les études en traductologie prennent rarement en considération, d'examiner les points d'achoppement que constitue l'interculturalité pour la traduction, et enfin de dégager quelques traits saillants qui résistent à la traduction et les raisons de leur intraduisibilité, objectifs qui ne peuvent être atteints sans réviser la tâche du traducteur face à l'altérité.

Enfin, dans «Le culturème Völkerwanderung et la question de l'historien traducteur ", Agnès Graceffa examine les problèmes de traduction auxquels se heurte la production historiographique grâce à l'exemple de la traduction du terme Völkerwanderung, qui est un « culturème » au sens où il renvoie à un référentiel propre à la culture allemande non partagé par la culture française, afin de montrer combien la réflexion traductologique sur l'étrangeté d'un terme et sa préservation éventuelle peut être utile à l'historien.

Quatre articles sont consacrés à la traduction littéraire, le premier ayant une dimension théorique, les trois autres se penchant sur la traduction d'un auteur ou d'une œuvre en particulier. Françoise Wuilmart aborde en effet la traduction littéraire d'un point de vue théorique et linguistique, dans « $\mathrm{La}$ traduction littéraire : source d'enrichissement de la langue d'accueil ». Elle revient sur l'éternelle question de la fidélité en traduction et propose de contourner l'alternative entre une traduction belle et infidèle ou laide mais infidèle en proposant de produire une "langue troisième ». Ainsi, dans le cas de la traduction vers le français, le traducteur devrait faire en sorte que le lecteur français « perçoive en filigrane, l'étrangeté du texte, ou plutôt son étrangéité » (p. 132). Wuilmart propose donc de faire en sorte que le français se laisse enrichir et féconder par d'autres langues, ce qui est la tâche de l'écrivain, mais aussi du traducteur, qui joue finalement un rôle capital.

C'est à la traduction de François Villon en espagnol qu'a choisi de s'intéresser Fernando Navarro Dominguez dans son article «Traduire le Moyen Âge : François Villon en espagnol ». Son objectif est de déterminer si le traducteur a su préserver 
l'historicité du texte par une réinvention créatrice, ce qui revient finalement à examiner comment a été rendue l'altérité du texte original. Il étudie douze différentes traductions, parues entre 1940 et 2002, ce qui lui permet d'esquisser un panorama de la traduction de Villon en espagnol et de montrer que presque tous les traducteurs ont privilégié le fond et ont parfois embelli le message au détriment de la forme.

On change d'époque avec le texte de Cindy LefebvreScodeller, qui étudie « Le traitement de la réduplication dans The Waves de Virginia Woolf ». Dans ce roman complexe, Virginia Woolf a utilisé à outrance la figure de la répétition, aussi bien au niveau lexical que phonétique. La réduplication est l'une des figures de répétition lexicale et consiste en la répétition d'un mot sans conjonction de coordination. L'objectif de cet article est d'étudier le traitement de la réduplication dans les deux traductions publiées à ce jour : celle de Marguerite Yourcenar (1931) et celle de Cécile Wajsbrot (1993), afin de déterminer s'il existe une différence réelle entre les choix des traductrices face à cette figure de répétition.

La problématique de l'intervention du traducteur dans les situations de conflit fait l'objet de l'article de Myriam SalamaCarr, "Normes et subjectivité - réflexions sur les traductions anglaise et française de Ra'aytu ramallah ", qui examine les traductions du récit autobiographique du poète et auteur Mourid Barghouti. Plus précisément, Salama-Carr cherche à examiner comment les notions de norme et de subjectivité en traduction peuvent nous aider à cerner les choix du traducteur, celui-ci jouant un rôle important dans la chaîne de domination politique, culturelle et idéologique induite par un conflit. L'article montre que ce récit, offrant un terrain privilégié pour l'étude des marqueurs de subjectivité, permet de jauger l'engagement du traducteur.

La traduction littéraire est un domaine qui donne plus de latitude à la subjectivité et à la créativité du traducteur. La créativité fait justement l'objet de plusieurs articles de La Traductologie dans tous ses états. John Gallagher examine ainsi « L'activité traduisante comme domaine de créativité ». Selon Gallagher, toute traduction, qu'elle soit littéraire ou non, comporte de nombreuses difficultés, 
qui ne peuvent être résolues que par la mise en œuvre d'une démarche créatrice : sur le plan lexical, sur les plan syntaxiques et paraphrastiques, qu'il examine successivement, à travers différents exemples. Il défend le point de vue selon lequel une approche purement rationnelle de la traduction doit être complétée par une approche intuitive et ludique, le traducteur devant posséder les qualités du créateur.

C'est aussi l'aspect créatif de la traduction, et le plaisir qui lui est associé, que Geneviève Hewson aborde dans son article «Entre désir et contrainte». Les nombreuses contraintes pesant sur le traducteur font souvent l'objet du discours traductologique, plutôt que "l'expérience fondamentalement jouissive de la traduction-écriture » (p. 117), qui est un acte potentiellement créatif. À en croire Hewson, même dans les situations les plus contraignantes, le traducteur a toujours un espace de liberté qui lui permet de faire un véritable travail de réécriture. Davantage, la contrainte en traduction est finalement l'élément qui, en déclenchant le dépassement, permet la création, qui est un acte jouissif.

L'aspect créateur de la traduction conçue comme réécriture est également l'objet de l'article de Henri Awaiss et Jarjoune Hardane. Les auteurs y traitent de leur ouvrage Eau de rose, eau de vinaigre, qui est « le fruit d'un acte de traduire à quatre mains » et retrace l'itinéraire de deux « vrais amis » (p. 136), qui écrivent et traduisent à deux. Écrit en arabe et en français, il mêle les thématiques Écrire, jouir, traduire et les modes d'écriture. Le traducteur y apparaît avant tout comme un médiateur, mais aussi un chef d'orchestre faisant jouer une partition inédite, tandis que la créativité est conçue comme l'élément commun à l'écriture et la traduction, à condition que les deux actes soient vécus comme un moment de bonheur.

Certains articles de l'ouvrage traitent d'aspects proprement linguistiques de la traduction. Ainsi, Corinne Wecksten se penche sur le problème des faux-amis en français et en anglais et les décalages connotatifs qu'une traduction littérale peut provoquer. En étudiant plus précisément les faux-amis partiels, Wecksten montre combien le problème des faux-amis est épineux dans le couple français-anglais, du fait de l'origine en 
partie commune des deux langues, ce qui nécessite beaucoup de vigilance de la part du traducteur.

Teresa Tomaskievicz se penche quant à elle sur les «Transferts de différents registres de la langue parlée », les registres de langue constituant un défi pour le traducteur. Tomaskieviz aborde différents contextes où un problème différent lié à la traduction des registres se pose : ainsi, la traduction de textes écrits imitant la langue parlée (dans le cadre de la traduction des dialogues romanesques de la littérature contemporaine), la traduction de textes écrits qui reproduisent différentes variantes dialectales (comme dans le cas de la traduction de la littérature acadienne - notamment le chiac - en polonais). Sont analysées ensuite la traduction orale des textes oraux dans le cadre des doublages de films et la traduction écrite de la langue parlée dans la traduction audiovisuelle sous forme de sous-titres.

Ce dernier article nous permet de faire le lien avec celui de Carmen Pineira-Tresmontant, qui traite lui aussi de la traduction au cinéma. Parmi les trois techniques de traduction au cinéma pour les mass-médias (le sous-titrage, le doublage ou la synchronisation et la voix-off), Pineira-Tresmontant choisit de s'intéresser au doublage, qui ressort de l'oral et qui est contraint par le temps, dans le cadre de la traduction du sitcom Friends. Le doublage est une technique difficile, car il faut se soucier de la synchronisation avec les mouvements des lèvres des acteurs. Dans le cas du doublage de Friends s'ajoute le problème de la traduction des jeux de mots, la série exprimant un certain humour américain qu'il faut tenter d'adapter à la culture du destinataire.

L'article de Jean Peeters ne rentre dans aucune des catégories abordées jusqu'ici, et s'annonce d'ailleurs comme marginal. Intitulé "Aux marges de la traductologie : Sur la réception de Harry Potter en France », cet article étudie la manière dont Harry Potter a été accueilli dans l'hexagone et comment des lecteurs très différents ont pu se retrouver dans le texte, et ce à travers l'analyse du courrier des lecteurs reçu aux éditions Gallimard, utilisé comme instrument de mesure de la réception. Cet article met en évidence le fait que la traductologie est tiraillée entre le vouloir dire de l'auteur d'une part et la compréhension 
qu'a le lecteur de la traduction d'autre part, car le traducteur opère bel et bien une interprétation de l'original.

Enfin, terminons avec l'article d'Anton Buena Garcia qui, ouvert sur l'avenir de la traduction, clôt adéquatement l'ouvrage. Dans «La traduction demain ", il rappelle, en citant Michel Ballard, que la recherche en traduction a acquis ses lettres de noblesse " parce qu'elle a fait un remarquable effort pour préciser ses objectifs et ses méthodes ", ce à quoi Michel Ballard a largement contribué. À l'heure actuelle, la mondialisation apporte un nouveau défi pour la traduction. Cet article cherche à entamer une réflexion sur l'avenir de la traduction en émettant un certain nombre d'hypothèses à la fois sur le devenir de la profession de traducteur, celui de sa formation et enfin celui de la théorie.

La Traductologie dans tous ses états est un recueil qui, par la variété des sujets abordés dans ses articles témoignant de la richesse actuelle de la réflexion et du dynamisme de la recherche en traductologie, s'avère être un bel hommage à Michel Ballard, chercheur qui a œuvré tout au long de sa carrière dans le sens d'un élargissement de l'horizon de sa discipline et de sa reconnaissance universitaire. Qu'il ait su inspirer autant de chercheurs de par le monde, dans le cadre de travaux et de méthodes aussi variés, atteste de l'impact de sa pensée dans l'univers de la traductologie.

\section{Stéphanie Roesler Université McGill}

\section{Dorothy Kenny et Kyongjoo Ryou, dirs. Across Boundaries: International Perspectives on Translation Studies, Newcastle, Cambridge Scholars Publishing, 2007, 240 p.}

À l'origine du présent ouvrage, il y a des communications données lors du premier colloque de l'International Association for Translation and Intercultural Studies (IATIS), tenu à Séoul en août 2004. Comme le titre l'indique, on y traverse des frontières, géographiques, certes (les auteurs sont originaires de douze pays différents), mais aussi linguistiques, culturelles, méthodologiques et disciplinaires. En réalité, on fait plus que les traverser, ces frontières : on les (re)négocie, on remet parfois leur existence en 